\section{Infant frontal asymmetry predicts child emotional availability}

\author{
Maria Licata, Markus Paulus, Nina Kühn-Popp, \\ Jorg Meinhardt and Beate Sodian
}

International Journal of Behavioral Development 2015, Vol. 39(6) 492-496

(C) The Author(s) 2015 Reprints and permissions: sagepub.co.uk/journalsPermissions.nav DOI: 10.1 I77/01650254I55768I6 ijbd.sagepub.com

(S)AGE

\begin{abstract}
While factors influencing maternal emotional availability (EA) have been well investigated, little is known about the development of child EA. The present longitudinal study investigated the role of frontal brain asymmetry in young children with regard to child EA (child responsiveness and involvement) in mother-child interaction in a sample of 28 children at 7,14 , and 50 months of age. When infants were 7 months of age, mother-child interaction quality was assessed using the EA-Scales. At 14 months, infants' resting asymmetric frontal activity was assessed by means of the electroencephalogram (EEG). When children were 50 months old, mother-child interaction quality was measured again. Analyses showed that relatively higher left frontal EEG activation was related to higher child involvement at 50 months, but not to child responsiveness. Those findings suggest a specific relation between individual differences in frontal asymmetry, and child approach and initiating behaviors in mother-child interaction.
\end{abstract}

\title{
Keywords
}

Mother-child interaction, emotional availability, EEG, frontal asymmetry

\section{Introduction}

Mother-child interaction quality plays a fundamental role in child development (Ainsworth, Blehar, Waters \& Wall, 1978; Bowlby, 1969). Theoretical accounts assume that a person's emotional availability (EA) (i.e. whether a person is emotionally accessible and responsive to the interaction partner), is a crucial indicator of interaction quality (e.g. Biringen \& Robinson, 1991; Emde, 1980), and is related to healthy child development (Biringen et al., 2005; Easterbrooks, Bureau \& Lyons-Ruth, 2012; Mäntymaa et al., 2009). A measure to assess emotional availability in interpersonal interactions is the Emotional Availability Scales (EAS, Biringen, 2008) which integrates attachment theory (Ainsworth et al., 1978; Bowlby, 1969) and perspectives focusing on emotions as a barometer of the parent-child relationship quality (Emde, 1980; Mahler, Pine \& Bergman, 1975). The EAS includes four maternal EA-dimensions (sensitivity, structuring, nonintrusiveness, and non-hostility), and two child EA-dimensions (responsiveness, and involvement). Maternal sensitivity refers to the mother's ability to respond in a warm, authentic and appropriate way to the child's signals; maternal structuring concerns the ability to scaffold the interaction; non-intrusiveness refers to the mother's tendency to let the child lead without interrupting it; and nonhostility is about the lack of hostile affect and behaviors (e.g. mocking the child, and rolling the eyes). Child responsiveness, in turn, refers to the child's tendency to respond to the mother in a positive, non-urgent way, whereas child involvement refers to the child's attempts to initiate interactions. EA is conceptualized as a dyadic construct, meaning that, for example, a mother cannot be highly sensitive if the child is not - to some extent - responsive, too.

Factors influencing maternal EA have been well investigated, indicating that it mainly varies as a function of maternal socio-emotional health (e.g. Biringen, Matheny, Bretherton,
Renouf \& Sherman, 2000; Easterbrooks, Biesecker \& Lyons-Ruth, 2000). However, the factors that affect child EA have been neglected. In the literature, the only factor that has been related to child EA is maternal EA (e.g. Bornstein et al., 2006; Kubicek, Riley, Coleman, Miller \& Linder, 2013). Since EA is conceptualized as a dyadic construct, it is obvious that maternal and child EA would be highly intercorrelated if assessed at the same time. However, longitudinal relations between the EA-dimensions also suggest that maternal sensitivity is predictive of child involvement and that maternal structuring predicts child responsiveness (Stack et al., 2012). Thus, the different EA-dimensions are related to each other both concurrently and longitudinally, with some dimensions being more strongly related to one another than others.

However, environmental variables such as maternal sensitivity are not the only factors that would be expected to influence child emotional availability. Theoretical accounts lead to the notion that endogenous, biological factors of the child might also be related to child behavior (see for example Crone \& Ridderinkhof, 2011). Interestingly, there is evidence that differences in frontal asymmetry in the electroencephalogram (EEG) are related to individual differences in social and emotional behavior (Fox, Schmidt, Calkins, Rubin \& Coplan, 1996; Paulus, Kühn-Popp, Licata, Sodian \& Meinhardt, 2013; Shankman et al., 2005; Smith \& Bell, 2010; for reviews see Harmon-Jones, Gable \& Peterson, 2010; Saby \& Marshall, 2012). For instance, in a study with 4-year-old children, Fox et al. (1995) showed that the disposition to respond with positive

Department of Psychology, Ludwig Maximilian University of Munich, Germany

\section{Corresponding author:}

Maria Licata, Leopoldstrasse, 13, Munich 80802, Germany.

Email: maria.licata@psy.Imu.de 
affect and approach behavior towards novel and stressful stimuli is associated with a relatively greater involvement of the left frontal cortex. In contrast, relatively higher right frontal activity has been related to negative affect and withdrawal (e.g. Cohen \& Shaver, 2004; Davidson \& Fox, 1989; for a meta-analysis, see Peltola et al., 2014). Moreover, adults with depression (e.g. Henriques \& Davidson, 1990) as well as infants of depressed mothers (e.g. Field, Diego, Hernandez-Reif, Schanberg \& Kuhn, 2002) showed greater relatively right frontal EEG activation. A study investigating adults revealed that individuals with insecure attachment showed lower left frontal asymmetry (i.e. greater right resting state activity) than securely attached individuals (Rognoni, Galati, Costa \& Crini, 2008).

Given the above-mentioned findings on the relations between frontal asymmetry and social behavior, and given the theoretical claims for biological influences on child EA, the present study explored the relations between individual differences in frontal asymmetry and child EA. More specifically, it investigated whether frontal asymmetry is related to both characteristics of child EA, or whether there are specific relations to the two child EA-dimensions. The present longitudinal study therefore aimed to investigate the role of the approach-avoidance system, assessed by frontal activation asymmetries in the EEG, with regard to child EA in motherchild interaction. To this end, maternal and child EA were assessed when children were 7 and 50 months old. As dimensions of child EA, child responsiveness and involvement were assessed. Maternal sensitivity as a control variable was included, since this EAdimension shows the highest correlation to child EA (e.g. Bornstein et al., 2006). Mother-child interaction was also assessed at 7 and 50 months. Within a period of 3 to 4 years, less temporal stability of EA can be assumed than over a shorter period of a few months (cf. Biringen et al., 2000; Bornstein et al., 2006; Lovas, 2005), but enough stability was expected in order to be able to detect significant correlations between the two measurement points (cf. Stack et al., 2012). Given the robust relationship between electrophysiological resting state patterns at 14 months and behavioral correlates in childhood, child EEG in a resting state at this age was assessed (cf. Cuevas \& Bell, 2011; Paulus et al., 2013; Shankman et al., 2005). It was hypothesized that greater left frontal activation would be related to higher child EA in mother-child interaction.

\section{Method}

\section{Participants}

Children and their mothers were part of a longitudinal study on early social-cognitive development (see Licata et al., 2014, for a further description of the sample). A subsample of 45 children participated in the current study. Eleven infants were not included in the final analyses due to fussiness during EEG testing, and six infants had to be excluded due to missing mother-child interaction data. The final sample of children that had valid data at all measurement points consisted of $n=28$ children ( 15 girls) and their mothers. The local ethics committee approved the study. Mean age of the children in the final sample was $\mathrm{M}=6.95$ months $(\mathrm{SD}=0.22$, range 6.53-7.33) at the first measurement point (t1), $\mathrm{M}=13.98$ months $(\mathrm{SD}=0.21$, range $=13.60-14.43)$ at the second appointment ( $\mathrm{t} 2)$, and $\mathrm{M}=50.55$ months $(\mathrm{SD}=0.67$, range 49.73 $52.80)$ at the third appointment ( $\mathrm{t} 3)$. In terms of maternal education, $28.6 \%(n=8)$ of the mothers had a not-college bound high-school degree (secondary school up to grade 10$), 17.9 \%(n=5)$ had a college-bound high-school degree (secondary school up to grade $13)$, and $53.6 \%(n=16)$ of the mothers had a college degree.

\section{Measures}

\section{Mother-child interaction quality}

Mother-child interaction quality was assessed in a laboratory setting using the Emotional Availability Scales (EAS, Biringen, 2008). Mothers were told to play with their child as they would usually do at home, sitting on a carpet with age-appropriate toys available. The interaction was videotaped and lasted for about 10 minutes.

The EA-Scales consist of six dimensions, four of them addressing the mother's side of EA and two the child's side of EA. All dimensions are rated on a 1- to 7-scale. In the present study, only the maternal sensitivity, maternal structuring, child responsiveness, and child involvement subscales were used. Maternal sensitivity focuses on genuine affect and emotional responsiveness to the child, but also comprises aspects like timing and flexibility in play. Child responsiveness assesses the degree to which the child reacts in a positive, non-urgent way to the mother, focusing on emotional rather than behavioral responsiveness as well as genuine, positive affect displayed by the child. Child involvement assesses the child's attempts to engage the mother in play in a non-urgent and relaxed way.

The coding was done by two trained coders. In order to assure reliability, $100 \%$ were rated by the first observer, and $35 \%$ of the videos $(n=11)$ were rated by the second observer. At 7 months of age, Cohen's Kappa was $\kappa=.89$ for maternal sensitivity, $\kappa=.88$ for child responsiveness, and $\kappa=.78$ for child involvement. At 50 months of age, Cohen's Kappa was $\kappa=.85$ for maternal sensitivity, $\kappa=.84$ for child responsiveness, and $\kappa=.89$ for child involvement.

\section{Electrophysiological assessment}

EEG was assessed at the age of 14-months. During the recording, the child sat quietly on his or her mother's lap and was presented with brightly colored bubbles on a computer screen (see for similar procedures Mundy, Card \& Fox, 2000; Paulus, Hunnius \& Bekkering, 2013). The recording lasted for at least 3 minutes and was stopped when the infant lost interest in the stimulus (indicated by strong motor activity or crying).

The EEG was continuously recorded from $17 \mathrm{Ag} / \mathrm{AgCl}$ active scalp electrodes (ActiCap, Brain Products, Germany) which were placed at standard positions according to the International 10/20 System (see for a similar electrode selection and position Cuevas, Raj \& Bell, 2012). The electrodes Fp1 and Fp2 were used to detect vertical eye movements and blinks, and F9 and F10 were used to detect horizontal eye-movements. The electrode $\mathrm{Cz}$ was used online as a reference electrode. Prior to EEG acquisition, electrode impedances were measured and accepted if they were below $10 \mathrm{k} \Omega$. Data were recorded with a Brain Amp amplifier (Brain Products, Gilching, Germany) and sampled at $500 \mathrm{~Hz}$; signals were bandpass filtered between 0.016 and $200 \mathrm{~Hz}$. Using the Vision Analyzer (Brain Products, Germany) a digital band pass filter of 1-20 Hz was applied offline and all electrodes were re-referenced to an average reference (see for a similar procedure Cuevas \& Bell, 2011; Marshall, Bar-Haim \& Fox, 2002). The EEG data were segmented into epochs of $1024 \mathrm{~ms}$ with $50 \%$ overlap. Segmented epochs were artifacted using semiautomatic and visual inspections and were excluded if an EEG channel exceeded $\pm 120 \mu \mathrm{V}$ or the epoch 
Table I. Descriptive statistics of the study variables.

\begin{tabular}{lrrr}
\hline$n=28$ & $M$ & SD & \multicolumn{1}{c}{ Range } \\
\hline Maternal Sensitivity (7 mo.) & 4.66 & 1.32 & $2.00-7.00$ \\
Child Responsiveness (7 mo.) & 4.93 & 1.31 & $2.50-7.00$ \\
Child Involvement (7 mo.) & 4.38 & 0.98 & $3.00-6.00$ \\
Child EEG Frontal Asymmetry (14 mo.) & -0.01 & 0.48 & $-1.40-0.97$ \\
Maternal Sensitivity (50 mo.) & 4.29 & 1.22 & $2.50-7.00$ \\
Child Responsiveness (50 mo.) & 4.45 & 1.17 & $2.50-7.00$ \\
Child Involvement (50 mo.) & 4.48 & 1.21 & $3.00-7.00$ \\
\hline
\end{tabular}

Note: high scores in sensitivity, responsiveness and involvement indicate high emotional availability; high EEG frontal asymmetry scores indicate greater left than right frontal EEG power.

contained eye movements, blinks or (motor) artifacts. The average number of usable segments was $294(\mathrm{SD}=111.8)$ epochs per infant (i.e. about $45 \%$ of all epochs were excluded from further analysis). A Hanning window was applied to artifact-free epochs and power spectra were computed via a Fast Fourier Transform. Spectral power, expressed as mean square microvolts $\left(\mu \mathrm{V}^{2}\right)$, was calculated for the 6- to $9-\mathrm{Hz}$ frequency band (which corresponds to the alpha frequency band for infants) and was used to compute asymmetries in activation (see for similar power band analyses Cuevas \& Bell, 2011; Reid, Striano \& Iacoboni, 2011). Frontal asymmetry scores (AsymF) were computed for each participant by subtracting the average log right power (F4, F8, F10) from the average log left power (F3, F7, F9). Based on the assumption that higher power indicates weaker cortical activation (see Fox et al., 1995, 1996; Saby \& Marshall, 2012), a positive score indicates more power and thus weaker activation at the left than the right site.

\section{Results}

All variables were normally distributed, with the lowest mean in EA child involvement and the highest mean in maternal sensitivity (both occurring at 7 months; see Table 1). Correlational analyses showed that almost all assessed EA-dimensions at both 7 and 50 months were highly intercorrelated. Moreover, the child EEG right frontal asymmetry score was negatively correlated with child involvement at 50 months, but not to the other EA-variables at 7 and 50 months of age (see Table 2).

In order to find out whether the relation between frontal asymmetry (AsymF) at 14 months and child involvement at 50 months was independent from early child EA or early or later maternal sensitivity, partial correlations were computed. The negative relation between relatively higher right than left frontal cortical activation and child involvement at 50 months remained significant even when controlling for maternal sensitivity, child responsiveness and involvement at 7 months, as well as maternal sensitivity and child responsiveness at 50 months $\left(r_{p a r}(21)=.53, p=.009\right)$. Moreover, in order to ensure that the relation between EEG alpha asymmetry and EA was specific to the frontal region, we conducted post-hoc correlations between EA and an alpha asymmetry score from temporal regions (AsymT: $\log$ T7-log T8). No significant correlations were found (all $r \mathrm{~s}<.13, p \mathrm{~s}>.54$ ).

\section{Discussion}

The present study is aimed at deepening our understanding of the development of child EA, namely child involvement and
Table 2. Correlations between the study variables.

\begin{tabular}{|c|c|c|c|c|c|c|c|}
\hline$n=28$ & I & 2 & 3 & 4 & 5 & 6 & 7 \\
\hline $\begin{array}{l}\text { I) Maternal Sensitivity } \\
7 \text { mo. }\end{array}$ & I & & & & & & \\
\hline $\begin{array}{l}\text { 2) Child Responsiveness } \\
7 \text { mo. }\end{array}$ & $.89 * * * *$ & I & & & & & \\
\hline $\begin{array}{l}\text { 3) Child Involvement } \\
7 \mathrm{mo} \text {. }\end{array}$ & $.85 * * *$ & $.89 * * *$ & I & & & & \\
\hline $\begin{array}{l}\text { 4) Child EEG Frontal } \\
\text { Asymmetry } 14 \mathrm{mo} .\end{array}$ & .17 & .21 & .18 & I & & & \\
\hline $\begin{array}{l}\text { 5) Maternal Sensitivity } \\
50 \mathrm{mo} .\end{array}$ & $.61^{* * * *}$ & $.56^{* * *}$ & $.59 * * *$ & -.08 & I & & \\
\hline $\begin{array}{l}\text { 6) Child Responsiveness } \\
50 \mathrm{mo} \text {. }\end{array}$ & $.53^{* *}$ & $.5 I^{* *}$ & $.51^{* *}$ & -.21 & $.94 * * *$ & I & \\
\hline $\begin{array}{l}\text { 7) C-Involvement } \\
50 \text { mo. }\end{array}$ & $.38^{*}$ & $.38^{*}$ & $.39 *$ & $-.39 *$ & $.84^{* * * *}$ & $.60^{* * * *}$ & * 1 \\
\hline
\end{tabular}

Note: ${ }^{* * *} p \leq .01,{ }^{* *} p \leq .0 \mathrm{I}, *_{p} \leq .05$, two-tailed.

responsiveness in mother-child interaction. More specifically, this is the first study that examined longitudinal relations between frontal EEG asymmetry as assessed in infancy and child EA in motherchild interaction at preschool age. Results showed that relatively higher left than right frontal cortical activation at 14 months was related to higher child involvement at 50 months. The relation remained stable even when controlling for maternal sensitivity and child responsiveness at 7 and 50 months, as well as child involvement at 7 months. With regard to child responsiveness, the EEG pattern showed no significant relation.

This is the first study to reveal the neural correlates of child interaction style with the mother. More specifically, the finding that child neural predisposition (as reflected by relatively higher left frontal EEG activation) turned out to be a predictor of child involvement independently from maternal sensitivity suggests that child involvement appears to be largely influenced by biological factors of the child. However, the finding that child responsiveness was strongly related to maternal sensitivity, but not to child frontal asymmetry, supports the assumption that the two facets of child EA - child responsiveness and involvement - reflect qualitatively distinct components of child interaction style (cf. Biringen, 2008). Child involvement as conceptualized by the EAS has a greater proactive component, referring to actively initiating interactions by approaching the mother and involving her into play, while child responsiveness rather refers to the child's reactions to maternal initiatives.

These findings extend previous findings associating relatively greater left frontal activation with high frequencies of initiating social interactions (e.g. Fox et al., 1995), as well as higher rates of empathic behaviors (Paulus et al., 2013) to the realm of mother-child interaction. This study supports the notion that individual differences in frontal asymmetry reflect a disposition of approach behavior and positive affect (cf. Harmon-Jones et al., 2010). Moreover, the findings of this study are in line with studies on adults, reporting significantly lower left frontal asymmetry in individuals with insecure attachment than in individuals with secure attachment (Rognoni et al., 2008).

Furthermore, these results are also informative for research on frontal resting state activation (Fox et al., 1995; Harmon-Jones et al., 2010) as they suggest that only the left-frontal asymmetry is specifically related to proactive behavioral tendencies, but not for 
reactive behaviors to other persons. Therefore, these findings add a piece to the puzzle of how the neural system impacts social functioning in mother-child-interaction. These initial findings suggest further research on how such differential neural activations develop from infancy to adulthood, and call for a deeper understanding of their structural and functional neural relationships across development (e.g. Crone \& Ridderinkhof, 2011). Moreover, it would be very interesting to investigate possible interaction effects between endogenous, neural factors and environmental influences with regard to the development of socio-emotional competencies.

The present study has also some limitations. First, due to the low sample size, causal modeling and tests for mediation were not possible. Thus, future research should replicate those findings with a larger sample size. Moreover, in the present study, the focus was on child predictors of child EA, whereas the only maternal variable included in the study was maternal EA. Thus, it is not known whether these results would hold up when controlling for maternal characteristics that are related to maternal EA (such as maternal attachment insecurity, and depression) (e.g. Easterbrooks et al., 2000). Future studies should investigate the relative impact of different maternal and child characteristics with regard to child EA. Moreover, further research should investigate whether certain moderator or mediator variables (e.g. child psychological adjustment, social competence, temperament, or also maternal characteristics, such as maternal depression) might affect the relation between relatively greater left frontal activity and child involvement in mother-child interaction. Despite these limitations, the current study is the first to demonstrate that developmental pathways differ for the two components of child EA (responsiveness and involvement), and that relatively higher left frontal cortical activation is longitudinally related to higher child involvement in mother-child interaction at preschool-age.

\section{Funding}

This research was funded by a grant from the German Research Council to Beate Sodian (DFG SO 213/27-1,2).

\section{References}

Ainsworth, M. D. S., Blehar, M. C., Waters, E., \& Wall, S. (1978). Patterns of attachment: A psychological study of the strange situation. Hillsdale, MI: Lawrence Erlbaum Associates.

Biringen, Z. (2008). The Emotional availability (EA) scales (4th edition). Unpublished manual, Colorado State University, Boulder, Colorado.

Biringen, Z., Matheny, A., Bretherton, I., Renouf, A., \& Sherman, M. (2000). Maternal representation of the self as parent: Connections with maternal sensitivity and maternal structuring. Attachment and Human Development, 2, 218-232.

Biringen, Z., Damon, J., Grigg, W., Mone, J., Pipp-Siegel, S., Skillern, S., \& Stratton, J. (2005). Emotional availability: Differential predictions to infant attachment and kindergarten adjustment based on observation time and context. Infant Mental Health Journal, 26, 295-308.

Biringen, Z., \& Robinson, J. L. (1991). Emotional availability: A reconceptualization for research. American Journal of Orthopsychiatry, 61, 258-271.

Bornstein, M. H., Gini, M., Putnick, D. L., Haynes, O. M., Painter, K. M., \& Suwalsky, J. T. D. (2006). Short-term reliability and continuity of emotional availability in infant-mother dyads across contexts of observation. Infancy, 10, 1-16.
Bowlby, J. (1969). Attachment and loss: attachment (Vol. 1). London: Hogarth Press.

Cohen, M. X., \& Shaver, P. R. (2004). Avoidant attachment and hemispheric lateralization of the processing of attachment- and emotion-related words. Cognition and Emotion, 18, 799-813.

Crone, E. A., \& Ridderinkhof, K. R. (2011). The developing brain: From theory to neuroimaging and back. Developmental Cognitive Neuroscience, 1, 101-109.

Cuevas, K., \& Bell, M. A. (2011). EEG and ECG from 5 to 10 months of age: Developmental changes in baseline activation and cognitive processing during a working memory task. International Journal of Psychophysiology, 80, 119-128.

Cuevas, K., Raj, V., \& Bell, M. A., (2012). Functional connectivity and infant spatial working memory: A frequency band analysis. Psychophysics 49, 271-280.

Davidson, R. A., \& Fox, N. A. (1989). Frontal brain asymmetry predicts infants' response to maternal separation. Journal of Abnormal Psychology, 98, 127-131.

Easterbrooks, M. A., Biesecker, G., \& Lyons-Ruth, K. (2000). Infancy predictors of emotional availability in middle childhood: The roles of attachment security and maternal depressive symptomatology. Attachment and Human Development, 2, 170-187.

Easterbrooks, M. A., Bureau, J.-F., \& Lyons-Ruth, K. (2012). Developmental correlates and predictors of emotional availability in mother-child interaction: A longitudinal study from infancy to middle childhood. Development and Psychopathology 24, 65-78.

Emde, R. N. (1980). Emotional availability: A reciprocal reward system for infants and parents with implications for prevention of psychosocial disorders. In P.M. Taylor (Ed.), Parent-infant relationships (pp. 87-115). Orlando, FL: Grune \& Stratton.

Field, T., Diego, M., Hernandez-Reif, M., Schanberg, S., \& Kuhn, C., (2002). Relative rightversus left frontal EEG in neonates. Developmental Psychobiology, 41, 147-155.

Fox, N. A., Rubin, K. H., Calkins, S. D., Marshall, T. R., Coplan, R. J., Porges, S. W., Long, J. M., \& Stewart, S. (1995). Frontal activation asymmetry and social competence at four years of age. Child Development, 66, 1770-1784.

Fox, N. A., Schmidt, L. A., Calkins, S. D., Rubin, K. H., \& Coplan, R. J. (1996). The role of frontal activation in the regulation and dysregulation of social behavior during the preschool years. Development and Psychopathology, 8, 89-102.

Harmon-Jones, E., Gable, P. A., \& Peterson, C. K. (2010). The role of asymmetric frontal cortical activity in emotion-related phenomena: A review and update. Biological Psychology, 84, 451-462.

Henriques, J. B., \& Davidson, R. J. (1990). Regional brain electrical asymmetries discriminate between previously depressed and healthy control subjects. Journal of Abnormal Psychology, 99, 22-31.

Kubicek, L. F., Riley, K., Coleman, J., Miller, G., \& Linder, T. (2013). Assessing the emotional quality of parent-child relationships involving young children with special needs: Applying the constructs of emotional availability and expressed emotion. Infant Mental Health Journal, 34, 242-256.

Licata, M., Paulus, M., Thoermer, C., Kristen, S., Woodward, A., \& Sodian, B. (2014). Mother-infant interaction quality and infants' ability to encode actions as goal-directed. Social Development, 23, 340-356.

Lovas, G. S. (2005). Gender and patterns of emotional availability in mother-toddler and father-toddler dyads. Infant Mental Health Journal, 26, 327-353.

Mäntymaa, M., Puuraa, K., Luoma, I., Vihtonen, V., Salmelinc, R. K., \& Tamminen, T. (2009). Child's behaviour in mother-child 
interaction predicts later emotional and behavioural problems. Infant and Child Development, 18, 455-467.

Mahler, M. S., Pine, F., \& Bergman, A. (1975). The psychological birth of the human infant Symbiosis and individuation. New York: Basic Books.

Marshall, P. J., Bar-Haim, Y., \& Fox, N. A. (2002). Development of the EEG from 5 months to 4 years of age. Clinical Neurophysiology, 113, 1199-1208.

Mundy, P., Card, J., \& Fox, N. A. (2000). EEG correlates of the development of infant joint attention skills. Developmental Psychobiology, 36, 325-338.

Paulus, M., Kühn-Popp, N., Licata, M., Sodian, B., \& Meinhardt, J. (2013). Neural correlates of prosocial behavior in infancy: Different neurophysiological mechanisms support the emergence of helping and comforting. NeuroImage, 66, 522-530.

Paulus, M., Hunnius, S., \& Bekkering, H. (2013). Neurocognitive mechanisms subserving social learning in infancy: Infants' neural processing of the effects of others' actions. Social Cognitive and Affective Neuroscience, 8, 774-779.

Peltola, M. J., Bakermans-Kranenburg, M. J., Alin, L. R. A., Huffmeijer, R., Biro, S., \& van Ijzendoorn, M. H. (2014). Resting frontal EEG asymmetry in children: Meta-analyses of the effects of psychosocial risk factors and associations with internalizing and externalizing behavior. Developmental Psychobiology, 56, $1377-1389$

Reid, V. M., Striano, T., \& Iacoboni, M. (2011). Neural correlates of dyadic interaction during infancy. Developmental Cognitive Neuroscience, 1, 124-130.

Rognoni, E., Galati, D., Costa, T., \& Crini, M. (2008). Relationship between adult attachment patterns, emotional experience and EEG frontal asymmetry. Personality and Individual Differences, 44, 909-920.

Saby, J. N., \& Marshall, P. J. (2012). The utility of EEG band power analysis in the study of infancy and early childhood. Developmental Neuropsychology, 37, 253-273.

Shankman, S. A., Tenke, C. E., Bruder, G. E., Durbin, C. E., Hayden, E. P., \& Klein, D. N. (2005). Low positive emotionality in young children: Association with EEG asymmetry. Developmental Psychopathology, 17, 85-98.

Smith, C. L., \& Bell, M. A. (2010). Infant frontal asymmetry as a predictor of toddlerhood internalizing and externalizing behaviors. Developmental Psychobiology, 52, 158-167.

Stack, D. M., Serbin, L. A., Girouard, N., Enns, L. N., Bentley, V. M. N., \& Schwartzman, A. E. (2012). The quality of mother-child relationship in high-risk dyads: An intergenerational, longitudinal study. Development and Psychopathology, 24, 93-105. 